\title{
Measuring the Effectiveness of Infrastructure-Level Detection of Large-Scale Botnets
}

\author{
Yuanyuan Zeng ${ }^{\dagger} \quad$ Guanhua Yan $^{\ddagger}$ \\ $\dagger$ University of Michigan \\ \{gracez, kgshin\}@eecs.umich.edu
}

\author{
Stephan Eidenbenz $^{\ddagger} \quad$ Kang G. Shin ${ }^{\dagger}$ \\ $\ddagger$ Los Alamos National Laboratory \\ \{ghyan, eidenben\}@lanl.gov
}

\begin{abstract}
Botnets are one of the most serious security threats to the Internet and its end users. In recent years, utilizing P2P as a Command and Control $(\mathrm{C \& C})$ protocol has become popular due to its decentralized nature that can help hide the botmaster's identity. Most bot detection approaches targeting P2P botnets either rely on behavior monitoring or traffic flow and packet analysis, requiring fine-grained information collected locally. This requirement limits the scale of detection. In this paper, we consider detection of $\mathrm{P} 2 \mathrm{P}$ botnets at a high-levelthe infrastructure level-by exploiting their structural properties from a graph analysis perspective. Using three different P2P overlay structures, we measure the effectiveness of detecting each structure at various locations (the Autonomous System (AS), the Point of Presence (PoP), and the router rendezvous) in the Internet infrastructure.
\end{abstract}

\section{INTRODUCTION}

A botnet consists of a group of coordinated compromised computers or bots that can mount attacks, such as Distributed Denial of Service (DDoS), spamming, phishing and identity theft. Botnets have become a serious security threat to the Internet users; they can bring down the entire system and disrupt Internet services. In a botnet, its Command and Control (C\&C) channel, in which the botmaster disseminates commands to, and get response from bots, is a key element. Traditional botnets utilize the IRC or HTTP protocol to implement centralized C\&C. Under this design, bots have to connect to central servers and even listen on certain channels to retrieve commands. Evidently, centralized $\mathrm{C} \& \mathrm{C}$ is vulnerable to a single point of failure, meaning that, whenever the central servers are identified and removed, the entire botnet will be disabled. To overcome this weakness, attackers have recently devised a decentralized $\mathrm{C} \& \mathrm{C}$ infrastructure exploiting the P2P protocol. A few noteworthy P2P botnets include Storm, Waledac and Conficker. Their P2P implementations are either based on an existing protocol (Storm utilized Kademila [12]) or completely customized.

The decentralized nature of P2P botnets inevitably makes their detection difficult. Approaches targeting centralized C\&C structures [7], [4] become ineffective under this new structure in which a botmaster can join, issue commands and leave at any time at any place. Generic detection approaches [6], [20] relying on behavior monitoring and traffic correlation analysis are mostly applicable at a small scale such as in edge networks and do not scale well because they require analysis of vast amounts of fine-grained information. In addition, if there are only a small number of bots in an edge network, detection based on bots' coordination may fail due to the limited number of instances seen. Given the fact that sizes of existing botnets are in the order of hundreds of thousands, effective and efficient large-scale detection needs to function at a high level without requiring fine-grained information that can only be obtained locally. As a P2P botnet has a structured overlay and connectivity patterns different from other applications from a graph analysis perspective, we naturally consider detection at the Internet infrastructure level by assessing the impact imposed by a P2P botnet at various network components and measuring the effectiveness of detection at such places.

In this paper, we evaluate the feasibility of detecting largescale P2P botnets with different network components in the Internet infrastructure. We construct three types of P2P-botnet overlays, map them to the corresponding AS (Autonomous System)-level underlays by inferring each overlay connection's AS-path, and thus determine the PoP (Point of Presence) path and geographical router rendezvous (co-located routers in the infrastructure) each connection goes through. We then take a close look at each individual AS, PoP and router rendezvous based on graph analysis. In particular, we calculate a few P2P traffic classification metrics to see whether the portion of botnet connections observed by a single network component can be identified as $\mathrm{P} 2 \mathrm{P}$ traffic. We would like to answer the following three questions from our analysis: (1) which network component is the best place for detection? (2) which P2P overlay structure can help hide the botnet traffic well? (3) what are the limitations of detection at the infrastructure level? Our main contribution lies in the thorough analysis of detection potential at the three infrastructure-level network components for three different $\mathrm{P} 2 \mathrm{P}$ overlay topologies. Our analysis has led to three key observations. First, a small number of ASes can observe almost all overlay connections, but the AS-level detection is less practical. PoPs can capture a large fraction of connections but the number of monitoring points is limited. Router rendezvous strike a balance between detection capability and feasibility. Second, a botnet has to make a tradeoff between resilience/efficiency and the ability to evade detection. Third, the infrastructure-level detection is not a panacea for all large-scale botnets: it needs to be integrated with detection 
schemes in edge networks to complete a detection picture.

The remainder of the paper is structured as follows. Section II describes related work. Section III details our methodology. Section IV presents our analysis results. The paper discusses a few challenges and concludes with Section V.

\section{RELATED WORK}

As botnets have become a major security threat, numerous approaches have been proposed for their detection and mitigation. Most of these approaches can only be applied to specific types of botnets, requiring in-depth understanding of the $\mathrm{C} \& \mathrm{C}$ profiles prior to their detection. A few generic approaches can detect different types of botnets regardless of the C\&C structure based on network packet and flow analysis [6] or combined host and flow analysis [20]. These approaches are effective for small-scale networks, such as in a campus or an enterprise network, but do not scale to large networks, because they need to obtain fine-grained information.

Considering the fact that $\mathrm{P} 2 \mathrm{P}$ botnets have structured overlay topologies, our approach takes a high-level view by exploiting structural properties derived from graph analysis, and is thus not limited by the availability of fine-grained information. In this regard, our work is closely related to graph-based traffic classification and analysis. Iliofotou et al. [10] proposed the use of Traffic Dispersion Graphs (TDGs) to monitor and classify network traffic. TDGs focus on networkwide interactions among hosts and show that graph features, such as the average degree and directionality, can be utilized to distinguish different applications. In our analysis, we adopt some of their metrics to determine whether the portion of traffic observed by a network component is P2P. BotGrep [14] analyzes structured graphs to locate bots by extracting P2P subgraphs from a communication graph containing background traffic. This approach was evaluated on entire botnet communication graphs and graphs with some edges removed. Unlike our work, BotGrep did not assess the botnet detection potential at different network components.

We are aware of two efforts on AS-level underlays mapped from P2P overlays. Rasti et al. [16] examined the global impact of the load imposed by a P2P overlay on the ASlevel underlay. Their focus was on the effect of overlay on the underlay, while our work is concerned with whether the effect can be utilized for detection. Jelasity et al. [11] constructed a modified Chord [17] topology and showed that the visibility of P2P botnet traffic at any single AS is limited and not sufficient for detection. Our work differs from theirs, as we have simulated three $\mathrm{P} 2 \mathrm{P}$ overlay topologies and observed the traffic not only at the AS-level but also at PoPs and router rendezvous, providing a more thorough analysis.

\section{Methodology}

\section{A. Overview}

We would like to achieve the following two goals. First, from a defender's perspective, we want to see how much of the botnet connections can be observed at a single network component and whether the respective communication graph has $\mathrm{P} 2 \mathrm{P}$ properties. Second, from an attacker's perspective, we want to study which $\mathrm{P} 2 \mathrm{P}$ overlay topology is stealthy enough so that at a single network component the graph-level information is not sufficient for detection. Our methodology consists of four main steps. In the first step, we construct a P2P overlay topology based on simulation and learn which end-device talks to which, i.e., the overlay connections. In the second step, to map the overlay to the AS-level underlay, we associate the IP addresses of the two end-devices of a connection with the corresponding ASes and calculate the ASlevel path between the two ASes. Given the AS paths, we then determine PoP-level paths and geographical router rendezvous paths. Knowing the paths of all connections, in the third step, we break down the connections on a per-AS, per-PoP, and perrouter-rendezvous basis. We are especially interested in the top ASes, PoPs and router rendezvous ranked by the number of connections going through. In the last step, we inspect those top network components individually. As in [11], [16], we do not consider background traffic but focus only on the traffic coming from the $\mathrm{P} 2 \mathrm{P}$ overlay, which is the best scenario, implying that if the P2P traffic cannot be identified under this situation, it will definitely not be captured when background traffic is present. We analyze several graph properties of the communication patterns at each top network component and determine whether it has the characteristics of $\mathrm{P} 2 \mathrm{P}$ traffic.

\section{B. Internet Infrastructure and End-Device Modeling}

Before detailing the four main steps, we briefly describe the Internet infrastructure and end-device modeling, which lays a basis for our methodology. We use multiple real-world datasets to construct a realistic model of the US Internet infrastructure. Table I lists all data sources in the model construction. In total, $73,884,296$ residential computers are generated in the entire US (except Hawaii and Alaska). The distribution of Internet access routers including dial-up, DSL and Cable is based on the market share of top US broadband companies and dialup service aggregators, and how these access routers connect to the backbone topology at Internet PoP locations is derived from AS peering relationships. We refer interested readers to [19] for details of this modeling.

\section{Overlay Topology Construction}

In recent years, $\mathrm{P} 2 \mathrm{P}$ overlays have become popular in botnet construction due to their decentralized nature. Many existing P2P overlays can be utilized to facilitate botnets' C\&C. We construct three types of P2P overlays: a widely-used Kademlia [12], a modified Chord [17] and a simple ring structure. We will later compare the structural properties of these three overlay topologies at each network component, the results of which will be presented in Section IV. Next, we will introduce each $\mathrm{P} 2 \mathrm{P}$ overlay followed by the way we construct the topology.

1) Kademlia: Kademlia is a Distributed-Hash-Table (DHT)-based P2P overlay protocol. Under this protocol, nodes are identified by node IDs and data items are identified by keys generated from a hash function; node IDs and keys 
TABLE I

DATA SOURCES USED IN OUR INTERNET INFRASTRUCTURE AND END-DEVICE MODEL

\begin{tabular}{|c|c|}
\hline Model component & Data sources \\
\hline Backbone topology & $\begin{array}{l}\text { Skitter dataset: http://www.caida.org/tools/measurement/skitter/ } \\
\text { Alias clustering data from the iPlane project: http://iplane.cs.washington.edu/data/alias_lists.txt } \\
\text { IP geolocation dataset: http://www.ip2location.com// }\end{array}$ \\
\hline Internet Point of Presence & Telegeography co-location database: http://www.telegeography.com/ \\
\hline Internet end-devices & US census data: census-block population in each $250 \times 250 \mathrm{~m}^{2}$ grid in US for a 24-hour duration [13] \\
\hline Internet access routers & $\begin{array}{l}\text { Dial-up service aggregators per each zip code: http://www.findanisp.com } \\
\text { Broadband ISP market share: http://www.leichtmanresearch.com/press/081108release.html } \\
\text { DSL central office locations: the LERG (Local Exchange Routing Guide) dataset from Telcordia } \\
\text { Cable company service locations: Dun \& Bradstreet (D\&B) dataset }\end{array}$ \\
\hline Internet routing & $\begin{array}{l}\text { BGP routing information from the University of Oregon Route Views Project: http://www.routeviews.org/ } \\
\text { AS prefix sets: http://www.fixedorbit.com/ } \\
\text { AS-level path inference: Qiu and Gao's algorithm [15] }\end{array}$ \\
\hline
\end{tabular}

are of the same length. Data items are stored in nodes whose IDs are close to data items' keys. The distance between two IDs, $X$ and $Y$, is calculated by bitwise exclusive or (XOR) operation: $X \oplus Y$. To search a data item, a node queries its neighbors for nodes whose IDs are close to this data item's key. After getting responses from its neighbors, the node continues to query those nodes that are closer to the key. This iterative process repeats until no closer nodes can be found. The benefit of Kademlia is its resilience to disruptions. Even if a few nodes are shut down or removed, the network will still be able to function. Kad network is an implementation of Kademlia. A few major P2P file sharing networks adopt the Kad implementation, such as Overnet and eMule. The Storm botnet was built upon Overnet.

An ideal way to construct the botnet overlay topology is to collect traffic traces from a real network, such as the Storm botnet. Since the Storm botnet is decentralized (i.e., there are no central venues where all communications can be observed), traces captured from the Storm botnet fall into two categories each of which has its drawbacks. In the first category, the traffic data were collected from a single or a few vantage points. They can only provide partial views of the botnet. In the second category, snapshots of the network were taken by network crawlers. The snapshots contain information such as which IPs are alive or dead, but cannot tell which IP connects to which IP. To characterize the effectiveness of detection at the underlay, a full picture capturing the entire network's connections is indispensable, so we have to construct a Kad network by simulation.

We use a high-fidelity botnet simulator BotSim [8] which integrates a popular P2P client named aMule [1], an implementation of Kad. Considering the fact that simulating a largescale botnet $(100,000$ bots) on a single or a few machines will take a prohibitively long time, our simulator was run on a distributed platform consisting of 400 machines, each with 2 Pentium III CPUs and 4Gb RAM. The simulator is a component of MIITS [18] which is built upon PRIME SSF [3], a distributed simulation engine. To make aMule work seamlessly on our simulator, several modifications were made to the original aMule code including intercepting time-related system calls and substituting them for simulated time function calls, and replacing socket API calls with network functions developed in MIITS. The rest of the code remains intact.
In a botnet, a majority of bots are compromised residential computers and not necessarily geographically close, so we have to take locations into account. Constrained by data availability, all bots in our simulation are in the US and their locations follow the geographical distribution of 73 million residential computers by state. The simulation of 100,000 bots executed for three days in simulation time. The output files log timestamps and connections in the network. We discarded the first day in which bots bootstrapped and the entire botnet stabilized, and kept the second and the third day for analysis. With the $\log$ files keeping track of which node talks to which other node and each node's state information, we need to obtain the IP address of each end-device to completely construct the overlay topology. For this, we randomly chose an end-device address from the state a bot resides in. This way, we created two Kad overlay topologies with 100,000 nodes, one day each.

2) Modified Chord: Chord is a DHT-based P2P protocol under which nodes form a ring structure. Each node has a predecessor and a successor and a few long range links. For example, there are a total of $N$ nodes in the ring. Node $i$ connects to nodes $(i-1) \bmod N$ and $(i+1) \bmod N$. It also connects to nodes $\left(i+2^{k}\right) \bmod N$ for $k=1,2, \ldots, \log _{2} N-1$ to form long-range links. In [11], modifications to Chord are proposed so that it is difficult to detect through graph analysis at any single AS. The main modification is to create clusters in the ring each of which has $\log _{2} N$ consecutive nodes. This way, nodes in the same cluster can share the same set of long-range links for routing. This topology is of interest to us because we want to see whether using a more realistic ASpath calculation algorithm can make a difference in detection and whether this topology can successfully hide itself at PoPs and router rendezvous as well. Since this modified Chord's topology is relatively simple, we constructed its overlay with 100,000 nodes directly based on its protocol without simulation. Following the same practice as in Kademlia, each enddevice address is a random draw from the state a bot belongs to.

3) Simple Ring: We also consider the simplest case: each node has only two neighbors-a predecessor and a successorto construct a ring structure. Presumably, this structure is stealthier and harder to detect than the modified Chord due to lack of connectivity at the overlay. We will verify this 
presumption in later analysis. Similar to the modified chord, this overlay has 100,000 nodes constructed directly and the bots' locations follow the same geographical distribution.

\section{Overlay to Underlay Mapping}

1) AS-Path: Given all overlay connections, the next step is to map each connection to an AS-level path. Note that each end-device IP address is associated with an AS number and determining an AS-path of a connection is actually to determine the AS-path between two ASes. We use the ASpath inference algorithm in [15] for inter-domain routing. The key idea is to infer AS paths from existing BGP routing tables.

2) PoP-Path: A PoP is an access point to the Internet. It is a physical location owned by an ISP or located at Internet exchange points and co-location centers. The computation of a PoP-level path is based on the respective AS-level path. Given a pair of source and destination end device IPs, the algorithm first determines the AS-level path $A S_{1} A S_{2} \ldots A S_{n}$, then iteratively finds the shortest IP-level path between PoPs connecting every neighboring pair of ASes and finally maps the IP-level path to the PoP-level path. We refer interested readers to [19] for details of this algorithm.

3) Router Rendezvous Path: Given an IP-level path of a connection, the geographical router rendezvous along this path can be determined.

\section{E. Traffic Breakdown}

Since our work focuses on structural properties of the communication graph observed by a single network component, not the entire botnet overlay per se, we need to break down the overlay connections on a per AS, per PoP and per router rendezvous basis. We then rank the three types of network components by the number of connections going through, and take a close look at the graph properties observed at each of the top 10 ASes, PoPs, and router rendezvous, respectively, in our analysis.

\section{F. Graph Analysis}

After breaking down the traffic, we know all connections that traverse a particular AS, PoP and router rendezvous. We can then generate directed graphs in which bots are represented by vertices and connections among them are represented by edges. For simplicity, all edges carry the same weight. Graph metrics to determine whether the traffic is $\mathrm{P} 2 \mathrm{P}$ are proposed in [9] and adopted to analyze the modified chord in [11]. In our analysis, we inspect the same set of features as in [11] for consistency. The features used to characterize P2P traffic include the number of weakly-connected components, size of the largest weakly-connected component, average node degree and $\mathrm{InO}$ (In Out) ratio. We introduce each of them as follows.

Number of Weakly-Connected Components: A weaklyconnected component is a maximal subgraph of a directed graph such that in the subgraph replacing all of its directed edges with undirected edges produces a connected undirected graph. For effective detection, we expect a small number of weakly-connected components. As one can imagine, a large number of connected components usually means small-size components that are less likely to exhibit typical P2P patterns.

Size of the Largest Weakly-Connected Component: This metric is meaningful to us because as pointed out in [10] the graph formed by a P2P network tends to be densely connected and have a large connected component including the majority of participating nodes.

Average Node Degree: This metric counts both the incoming and outgoing edges of a node, i.e., ignoring the directionality. A graph with a high average degree tends to be tightly-connected and P2P networks normally have high average node degrees.

InO Ratio: The metric calculates the percentage of nodes in the graph that have both incoming and outgoing edges. This metric is of interest because under client-server protocols such as HTTP and SMTP, clients usually initiate connections (outgoing edges) whereas servers normally accept connections (incoming edges). But nodes in P2P networks usually serve as both clients and servers so that $\mathrm{P} 2 \mathrm{P}$ 's $\mathrm{InO}$ is distinctively higher than others.

\section{AnAlysis Results}

This section presents our analysis results. After constructing three different P2P overlay topologies, namely, Kad, the modified Chord and the simple ring, we examine their communication graphs at three types of network components. We conduct a graph analysis first at the AS-level, then the PoP-level and finally, the router-rendezvous-level, and show the graph features at the top 10 places of each level.

\section{A. AS-Level Analysis}

We first take a look at the AS-level graphs of three different topologies. Table II shows the Kad graph properties for day1 at top 10 ASes (for brevity we omit day2's result as they are similar to day1's), ranked by the number of unique connections going through. We map the AS numbers to ISPs using the AS-name lookup list [2]. Note that the traffic percentage at a single AS is calculated by the number of unique connections observed at that particular AS divided by the total number of unique connections in the entire overlay topology. Since one connection usually can be seen at more than one AS (this is why the first column of the table adds up to more than 100\%), we count each connection only once while calculating the number of connections observed at multiple ASes altogether. Following such calculations, in day 1, top 10 ASes aggregated together can observe $98.95 \%$-almost all of the Kad overlay's unique connections. In particular, the top 1 AS (3356/Level3) alone can see two thirds of the overlay connections with all nodes (100000) in the picture. Even for ASes carrying fewer connections, they have at least 99937 nodes' connections traverse through. Most importantly, at each top AS, all nodes are weakly-connected with each other, forming one giant weakly-connected component. This property can facilitate detection because one single weakly-connected graph containing a majority of connections is more likely to demonstrate P2P characteristics and easier to get caught than 
TABLE II

KAD AS-LEVEL

\begin{tabular}{cccccccc}
\hline $\begin{array}{c}\text { Kad Day1 } \\
\text { ISP }\end{array}$ & AS & Percentage & \# of Nodes & \# of Edges & Avg Degree & \# of Conn Comp & InO \\
\hline Level3 & 3356 & $65.25 \%$ & 100000 & 38192566 & 763.9 & 1 & $99.02 \%$ \\
AT\&T & 7018 & $35.33 \%$ & 100000 & 20679083 & 413.6 & 1 & $99.02 \%$ \\
XO & 2828 & $23.39 \%$ & 100000 & 13691127 & 273.8 & 1 & $99.02 \%$ \\
Sprint & 1239 & $8.32 \%$ & 99983 & 4872140 & 97.5 & 1 & $99.01 \%$ \\
Verizon & 19262 & $8.30 \%$ & 100000 & 4859686 & 97.2 & 1 & $100.00 \%$ \\
Qwest & 209 & $8.28 \%$ & 100000 & 4848724 & 97.0 & 1 & $99.02 \%$ \\
NTT & 2914 & $7.78 \%$ & 99993 & 4556302 & 91.1 & 1 & $99.02 \%$ \\
BellSouth & 6389 & $7.78 \%$ & 100000 & 4554972 & 91.1 & 1 & $99.01 \%$ \\
AT\&T & 7132 & $6.78 \%$ & 99995 & 3965587 & 79.3 & 1 & $100.00 \%$ \\
UUNET & 701 & $5.38 \%$ & 99937 & 3148400 & 63.0 & 1 & $88.13 \%$ \\
\hline
\end{tabular}

TABLE III

MODIFIED CHORD AS-LEVEL

\begin{tabular}{cccccccc}
\hline ISP & AS & Percentage & \# of Nodes & \# of Edges & Avg Degree & \# of Conn Comp & InO \\
\hline Level3 & 3356 & $64.25 \%$ & 80620 & 112431 & 2.8 & 9639 & $66.22 \%$ \\
AT\&T & 7018 & $38.09 \%$ & 54272 & 66650 & 2.5 & 10534 & $51.62 \%$ \\
XO & 2828 & $22.73 \%$ & 36234 & 39784 & 2.2 & 7470 & $47.03 \%$ \\
Verizon & 19262 & $9.43 \%$ & 17365 & 16494 & 1.9 & 3726 & $37.01 \%$ \\
NTT & 2914 & $8.09 \%$ & 15339 & 14151 & 1.8 & 3384 & $34.45 \%$ \\
Sprint & 1239 & $7.64 \%$ & 14908 & 13366 & 1.8 & 3602 & $31.16 \%$ \\
Qwest & 209 & $7.20 \%$ & 14642 & 12594 & 1.7 & 3757 & $27.99 \%$ \\
AT\&T & 7132 & $7.13 \%$ & 13849 & 12482 & 1.8 & 2956 & $33.29 \%$ \\
BellSouth & 6389 & $6.82 \%$ & 13486 & 11934 & 1.8 & 3080 & $30.47 \%$ \\
UUNET & 701 & $6.27 \%$ & 13900 & 10978 & 1.6 & 4305 & $16.41 \%$ \\
\hline
\end{tabular}

TABLE IV

SIMPLE RING AS-LEVEL

\begin{tabular}{cccccccc}
\hline ISP & AS & Percentage & \# of Nodes & \# of Edges & Avg Degree & \# of Conn Comp & InO \\
\hline Level3 & 3356 & $64.76 \%$ & 79327 & 64755 & 1.6 & 14522 & $63.31 \%$ \\
AT\&T & 7018 & $37.51 \%$ & 51316 & 37511 & 1.5 & 13805 & $46.20 \%$ \\
XO & 2828 & $22.81 \%$ & 32148 & 22805 & 1.4 & 9343 & $41.88 \%$ \\
Verizon & 19262 & $9.30 \%$ & 13632 & 9297 & 1.3 & 4335 & $36.40 \%$ \\
NTT & 2914 & $8.05 \%$ & 11867 & 8046 & 1.3 & 3821 & $35.60 \%$ \\
Sprint & 1239 & $7.53 \%$ & 11604 & 7532 & 1.3 & 4072 & $29.82 \%$ \\
Qwest & 209 & $7.36 \%$ & 11494 & 7362 & 1.3 & 4132 & $28.10 \%$ \\
AT\&T & 7132 & $7.07 \%$ & 10430 & 7066 & 1.3 & 3364 & $35.49 \%$ \\
BellSouth & 6389 & $6.73 \%$ & 10193 & 6728 & 1.3 & 3465 & $32.01 \%$ \\
UUNET & 701 & $6.17 \%$ & 10831 & 6166 & 1.1 & 4665 & $13.86 \%$ \\
\hline
\end{tabular}

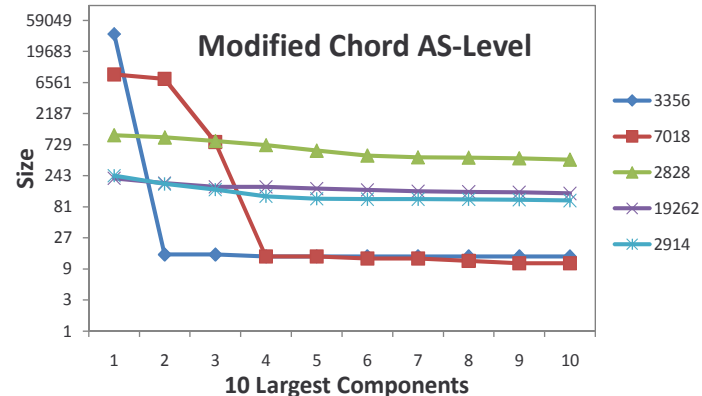

Fig. 1. Modified Chord: 10 largest components at top 5 ASes

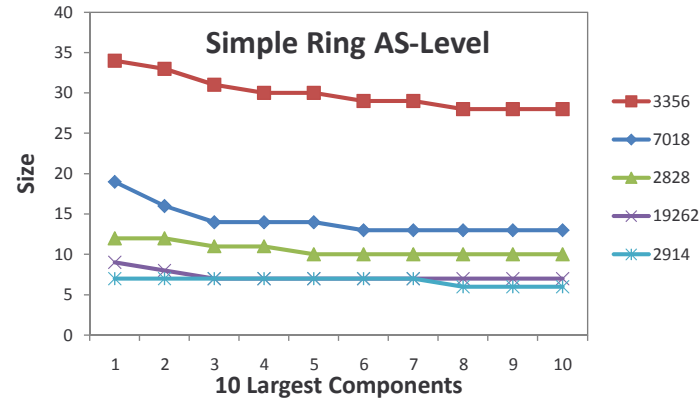

Fig. 2. Simple ring: 10 largest components at top 5 ASes 
a disconnected graph with many connected components of small sizes. As suggested in [9], two metrics can characterize $\mathrm{P} 2 \mathrm{P}$ traffic. One is a high average degree (larger than 2.8), and the other is a high $\mathrm{InO}$ ratio (large than 1\%). At all top ASes, the average degrees and $\mathrm{InO}$ values are high enough for P2P classification: the lowest value of average degree is 63.0 and that of $\mathrm{InO}$ is $88.13 \%$. Thus, all top AS venues have high visibility of Kad's overlay that demonstrates typical P2P patterns, sufficient for detection.

Table III shows graph features of the modified Chord at top 10 ASes. Compared to Kad, top 10 AS numbers remain same but their ranks change a bit. They in total observe $99.61 \%$, an enormous fraction of overlay connections and the top 1 AS is still 3356 witnessing $64.25 \%$ of all connections. Note that the AS observing the most can see 80620 while the one observing the least can only see 13900 nodes. As for the number of connected components, to the contrary of $\mathrm{Kad}$, each AS's graph is not well connected and has thousands of connected components. Figure 1 shows in log scale the sizes of 10 largest weakly-connected components at top 5 ASes. Top 1 AS 3356's largest component has 36532 nodes but all other components are very small containing 15 nodes or so. Top 2 AS 7018 has two large components with 8729 and 7506 nodes respectively and sizes of other components drop significantly. The component sizes remain stable at other ASes, all in the order of hundreds. Due to the relatively sparse structure of the modified Chord, unsurprisingly, the average degree at each AS is low-from 2.8 to 1.6 , though the $\mathrm{InO}$ values are highfrom $66.22 \%$ to $16.41 \%$. Taking all metrics into account, AS 3356 is able to detect the $\mathrm{P} 2 \mathrm{P}$ overlay since it can see a large portion of the overlay with typical $\mathrm{P} 2 \mathrm{P}$ patterns, if not the entire one. If we relax the average degree threshold a bit, AS 7018 may also be a good venue to make detection efforts considering the two large connected components. We think it is hard for the rest of the ASes to do so due to their relatively fragmented views. Note that our observations on the modified Chord are slightly different from those in [11] which concludes that even at the most central (top) ASes the average degrees are less than 2 and connected components are mostly of size 2 and 3 with the maximal containing 29 nodes. This difference may be attributed to the way of mapping the overlay to the underlay: they make the number of overlay nodes in each AS proportional to the size of the AS whereas we consider the geographical distribution of nodes. In addition, our AS-path inference algorithm is also different from theirs: they assume shortest paths while our AS-pathes are derived from real-world BGP routing tables.

When it comes to the simple ring structure (Table IV), the top AS numbers do not change, and their ranks are the same as those for the modified Chord. $99.62 \%$ of overlay connections traverse through top 10 ASes. Though the top 1 AS 3356 can see $64.76 \%$ of overlay connections, the number of nodes visible (79327) are more than the number of edges (64755), resulting in a great number of connected components (14522) and small component sizes. As seen in Figure 2, 3356's largest component only has 34 nodes. We also verify that a majority of
3356 connected components have fewer than 10 nodes. The average degrees are all below 2, which is expected because each node only has a predecessor and a successor so that the average degree of the entire graph is only 2. Even though the InO values are high enough, detection based on scattered information at a single AS is difficult.

\section{B. PoP-Level Analysis}

At the PoP level, we also present graph features at each top PoP of three P2P structures. PoPs are represented by ID numbers and ranked by the number of unique connections going through as well. In Kad's case (Table V), the top 10 PoPs account for $80.88 \%$ of overlay connections, a slight drop compared to that observed at top 10 ASes which can see more than $98 \%$. This makes sense because PoPs, normally as traffic exchange points, are not able to see intra-domain traffic taking place within ASes. The top PoP 74 alone is able to observe $53.78 \%$ of all connections. Similar to the AS-level, not only almost all nodes (more than 99975) can be seen at each top PoP, but also they are weakly connected forming one single component. The average degrees and $\mathrm{InO}$ ratios are well above the P2P classification thresholds.

In the case of the modified Chord (Table VI), top PoPs are almost the same as those of Kad and only the ranks change, taking up $80.29 \%$ of overlay connections aggregately. 74 is still the top 1 PoP observing $54.07 \%$ of total connections containing 77488 nodes, but all other PoPs observe fewer than 20000 nodes. As for sizes of weakly connected components, shown in Figure 3 in log scale, PoP 74's largest component is of size 23153 and others are quite small. Other PoPs' component sizes are fewer than 300. Given all these statistics, if the average degree threshold can be relaxed a bit, PoP 74 can be a good place for detection.

In the case of the simple ring (Table VII), the PoP numbers are exactly the same as those of modified Chord. $89.25 \%$ of overlay connections reach top 10 PoPs with $54.51 \%$ traversing PoP 74. Despite the fact that half of overlay connections can be observed at PoP 74, similar to the AS-Level, the number of edges is smaller than the number of nodes. The largest component of PoP 74 is very small containing 22 nodes (Figure 4). It is the same case for all other top PoPs. Though InO values are moderate, low average degrees and a good many small connected components can prevent the P2P structure from being captured at any PoP.

\section{Router-Rendezvous-Level Analysis}

At the router-rendezvous level, we present results in the same way. Router rendezvous are denoted by ID numbers and ordered by the number of unique overlay connections observed. For the Kad structure, as shown in Table VIII, the top 10 router rendezvous see $89.75 \%$ of total connections. The top 1 router rendezvous number 2 is reached by $68.77 \%$ of all connections. A majority of nodes (more than 98858) appear in the graph as one giant component at each top router rendezvous. In addition, high average degrees and $\mathrm{InO}$ values make detection feasible. Let us take a look at the 
TABLE V

KAD PoP-LEVEL

\begin{tabular}{ccccccc}
\hline $\begin{array}{c}\text { Kad Day1 } \\
\text { PoP }\end{array}$ & Percentage & \# of Nodes & \# of Edges & Avg Degree & \# of Conn Comp & InO \\
\hline 74 & $53.78 \%$ & 100000 & 31479094 & 629.6 & 1 & $100.00 \%$ \\
7 & $10.29 \%$ & 100000 & 6024939 & 120.5 & 1 & $99.94 \%$ \\
435 & $8.27 \%$ & 100000 & 4837622 & 96.8 & 1 & $98.50 \%$ \\
11 & $8.14 \%$ & 99998 & 4763870 & 95.3 & 1 & $99.86 \%$ \\
128 & $7.77 \%$ & 99981 & 4550316 & 91.0 & 1 & $99.52 \%$ \\
282 & $7.37 \%$ & 99995 & 4315967 & 86.3 & 1 & $100.00 \%$ \\
4 & $7.27 \%$ & 99977 & 4257513 & 85.2 & 1 & $99.73 \%$ \\
267 & $6.72 \%$ & 99992 & 3934199 & 78.7 & 1 & $100.00 \%$ \\
291 & $6.26 \%$ & 99975 & 3661420 & 73.2 & 1 & $100.00 \%$ \\
295 & $6.25 \%$ & 99997 & 3658911 & 73.2 & 1 & $99.97 \%$ \\
\hline
\end{tabular}

TABLE VI

MODIFIED CHORD POP-LEVEL

\begin{tabular}{ccccccc}
\hline PoP & Percentage & \# of Nodes & \# of Edges & Avg Degree & \# of Conn Comp & InO \\
\hline 74 & $54.07 \%$ & 77488 & 94629 & 2.4 & 16735 & $48.00 \%$ \\
7 & $9.27 \%$ & 19927 & 16222 & 1.6 & 6095 & $21.91 \%$ \\
267 & $7.99 \%$ & 14764 & 13981 & 1.9 & 3092 & $34.80 \%$ \\
11 & $7.98 \%$ & 17225 & 13957 & 1.6 & 5334 & $18.75 \%$ \\
128 & $7.46 \%$ & 17169 & 13058 & 1.5 & 5673 & $17.39 \%$ \\
4 & $7.25 \%$ & 15962 & 12686 & 1.6 & 4834 & $20.36 \%$ \\
435 & $6.94 \%$ & 13649 & 12151 & 1.8 & 3067 & $32.38 \%$ \\
282 & $6.81 \%$ & 13677 & 11913 & 1.7 & 3184 & $31.41 \%$ \\
291 & $6.36 \%$ & 12433 & 11137 & 1.8 & 2683 & $32.68 \%$ \\
295 & $5.84 \%$ & 11877 & 10228 & 1.7 & 2803 & $29.32 \%$ \\
\hline
\end{tabular}

TABLE VII

SIMPLE RING PoP-LEVEL

\begin{tabular}{ccccccc}
\hline PoP & Percentage & \# of Nodes & \# of Edges & Avg Degree & \# of Conn Comp & InO \\
\hline 74 & $54.51 \%$ & 75999 & 54506 & 1.4 & 21493 & $43.44 \%$ \\
7 & $9.40 \%$ & 16165 & 9400 & 1.2 & 6765 & $16.30 \%$ \\
11 & $7.78 \%$ & 13648 & 7779 & 1.1 & 5869 & $13.99 \%$ \\
128 & $7.63 \%$ & 13765 & 7631 & 1.1 & 6134 & $10.88 \%$ \\
267 & $7.52 \%$ & 11079 & 7521 & 1.4 & 3558 & $35.77 \%$ \\
4 & $7.31 \%$ & 12505 & 7305 & 1.2 & 5200 & $16.83 \%$ \\
435 & $7.13 \%$ & 10568 & 7127 & 1.3 & 3441 & $34.88 \%$ \\
282 & $7.08 \%$ & 10587 & 7078 & 1.3 & 3509 & $33.71 \%$ \\
291 & $6.37 \%$ & 9392 & 6373 & 1.4 & 3019 & $35.71 \%$ \\
295 & $5.77 \%$ & 8829 & 5774 & 1.3 & 3055 & $30.80 \%$ \\
\hline
\end{tabular}

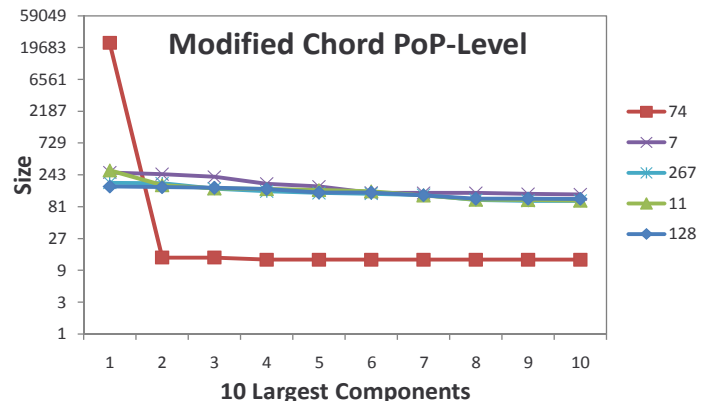

Fig. 3. Modified Chord: 10 largest components at top 5 PoPs

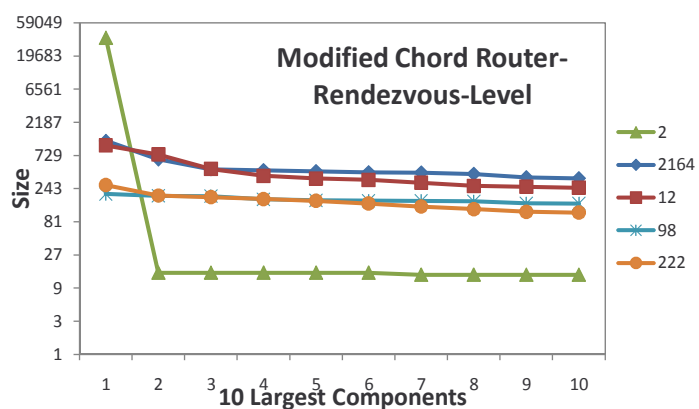

Fig. 5. Modified Chord: 10 largest components at top 5 locations

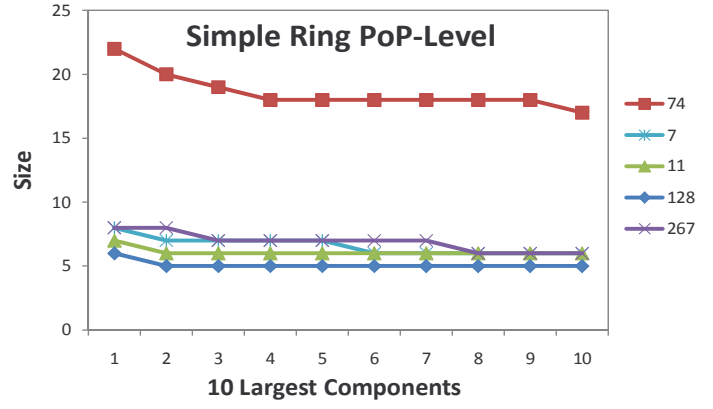

Fig. 4. Simple ring: 10 largest components at top 5 PoPs

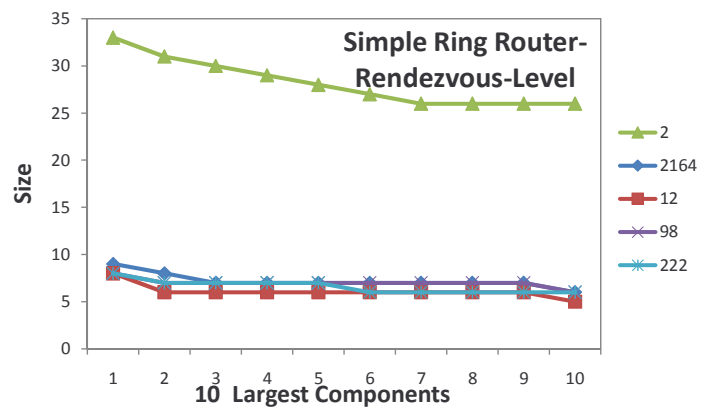

Fig. 6. Simple ring: 10 largest components at top 5 locations 
TABLE VIII

KAD ROUTER-RENDEZVOUS-LEVEL

\begin{tabular}{ccccccc}
\hline $\begin{array}{c}\text { Kad Day1 } \\
\text { Router }\end{array}$ & Percentage & \# of Nodes & \# of Edges & Avg Degree & \# of Conn Comp & InO \\
\hline 2 & $68.77 \%$ & 100000 & 40251799 & 805.0 & 1 & $100.00 \%$ \\
2164 & $14.91 \%$ & 99959 & 8728267 & 174.6 & 1 & $98.96 \%$ \\
12 & $11.90 \%$ & 99997 & 6967203 & 139.3 & 1 & $84.22 \%$ \\
98 & $11.75 \%$ & 100000 & 6874621 & 137.5 & 1 & $100.00 \%$ \\
222 & $9.26 \%$ & 100000 & 5419174 & 108.4 & 1 & $99.99 \%$ \\
8919 & $8.30 \%$ & 100000 & 4855632 & 97.1 & 1 & $98.50 \%$ \\
745 & $7.82 \%$ & 99997 & 4579803 & 91.6 & 1 & $99.85 \%$ \\
82 & $7.33 \%$ & 99978 & 4288889 & 85.8 & 1 & $99.74 \%$ \\
47 & $6.99 \%$ & 98858 & 4090556 & 82.8 & 1 & $92.32 \%$ \\
88 & $6.67 \%$ & 99997 & 3904395 & 78.1 & 1 & $99.71 \%$ \\
\hline
\end{tabular}

TABLE IX

MODIFIED CHORD ROUTER-RENDEZVOUS-LEVEL

\begin{tabular}{ccccccc}
\hline Router & Percentage & \# of Nodes & \# of Edges & Avg Degree & \# of Conn Comp & InO \\
\hline 2 & $68.76 \%$ & 88913 & 120337 & 2.7 & 13816 & $59.33 \%$ \\
2164 & $15.00 \%$ & 29299 & 26245 & 1.8 & 8964 & $25.60 \%$ \\
12 & $11.57 \%$ & 23682 & 20247 & 1.7 & 7629 & $20.07 \%$ \\
98 & $11.33 \%$ & 21641 & 19821 & 1.8 & 5586 & $31.07 \%$ \\
222 & $8.73 \%$ & 17779 & 15280 & 1.7 & 4771 & $27.68 \%$ \\
745 & $7.59 \%$ & 16673 & 13275 & 1.6 & 5286 & $17.14 \%$ \\
82 & $7.29 \%$ & 16133 & 12758 & 1.6 & 4926 & $19.98 \%$ \\
8919 & $6.94 \%$ & 13649 & 12151 & 1.8 & 3067 & $32.38 \%$ \\
88 & $6.26 \%$ & 12913 & 10962 & 1.7 & 3364 & $25.96 \%$ \\
57 & $6.16 \%$ & 13606 & 10784 & 1.6 & 4029 & $19.42 \%$ \\
\hline
\end{tabular}

TABLE $X$

SIMPLE RING ROUTER-RENDEZVOUS-LEVEL

\begin{tabular}{ccccccc}
\hline Router & Percentage & \# of Nodes & \# of Edges & Avg Degree & \# of Conn Comp & InO \\
\hline 2 & $68.89 \%$ & 88161 & 68885 & 1.6 & 19276 & $56.27 \%$ \\
2164 & $15.12 \%$ & 25513 & 15122 & 1.2 & 10391 & $18.54 \%$ \\
12 & $11.35 \%$ & 20126 & 11351 & 1.1 & 8775 & $12.80 \%$ \\
98 & $11.28 \%$ & 17720 & 11275 & 1.3 & 6445 & $27.26 \%$ \\
222 & $8.93 \%$ & 14243 & 8933 & 1.3 & 5310 & $25.44 \%$ \\
745 & $7.42 \%$ & 13218 & 7419 & 1.1 & 5799 & $12.26 \%$ \\
82 & $7.36 \%$ & 12653 & 7356 & 1.2 & 5297 & $16.27 \%$ \\
8919 & $7.13 \%$ & 10568 & 7127 & 1.3 & 3441 & $34.88 \%$ \\
88 & $6.10 \%$ & 9762 & 6102 & 1.3 & 3660 & $25.02 \%$ \\
47 & $6.06 \%$ & 9669 & 6061 & 1.3 & 3608 & $25.37 \%$ \\
\hline
\end{tabular}

modified Chord at the router-rendezvous level (Table IX). There is one new router rendezvous in the top 10 list that does not appear in that of Kad's and the ranks of the two lists are quite similar. Top 10 router rendezvous carry $89.96 \%$ of total connections and the top 1 router rendezvous is still 2 accounting for $68.76 \%$ of connections including 88913 nodes. As for the sizes of weakly connected components, the trend does not differ much from that at the AS- and PoP-level. The top 1 router rendezvous's largest connected component is of a big size-35943 nodes (Figure 5 in log scale) and other components have small sizes (fewer than 15 nodes). With a distinctive average degree and a high InO value, this router rendezvous is a reasonable venue for capturing the modified Chord. Finally, for the simple ring structure (Table X), the top router rendezvous list is the same as that of Kad. Top 10 router rendezvous observe $80.54 \%$ of overlay connections and router 2 sees $68.89 \%$ of them. With more nodes than edges at each top router rendezvous, it is difficult to get a good view of the overlay. Similar to AS- and PoP-level, the top 1 router rendezvous's largest component contains 33 nodes.
The average degrees are unsurprisingly low, insufficient for detection.

\section{Insights from Analysis}

From the above analysis, we have several key observations. First, the visibility of Kad's overlay and structure at the top places of all levels is good enough for detection; the modified Chord's P2P characteristics can be captured by a few top locations but not all; and the information of the hypothetical simple ring's topology at all levels is quite fragmented and hardly useful for detection. From the attacker's viewpoint, in terms of efficiency, Kad has the most efficient routing: contacting $O(\log N)$ nodes during a search (where $N$ is the size of the network); the modified Chord can achieve $O\left(\log ^{2} N\right)$ hops; and the simple ring is the worst, requiring $O(N)$ steps. From resilience's perspective, the Kad network is shown to be robust to a few types of mitigation strategies such as cutting off random nodes and removing peers learnt from bots' peer lists [5]; the simple ring structure is evidently fragileremoving a couple of nodes can disconnect the overlay; and 
the modified Chord structure hits the middle ground: not as resilient as Kad but better than the simple ring. We believe that, while constructing a P2P botnet, the attacker needs to strike a balance between resilience or efficiency and the ability to evade detection. Although the simple ring can hide its traffic well at various network components, to build upon this structure the botnet has to compromise resilience and $\mathrm{C} \& \mathrm{C}$ efficiency. The modified Chord makes a tradeoff though its structural properties cannot be concealed at some locations. Kad was successfully utilized by the Storm botnet, but given our detection strategy, to use it for a future botnet, the attacker has to come up with techniques to mask its P2P patterns.

Second, from detection's perspective, AS-level provides better overlay views than PoP- and router-rendezvous-level do, but is less practical than the other two for actual detection deployment. Since AS is only a logical concept, capturing all connections within one AS requires collaboration and synchronization among multiple physical devices at different geographical locations, which renders it highly impractical. From our analysis, we can see that at the PoP-level, detecting Kad and the modified Chord is very likely though the latter is only visible to the top 1 PoP. Compared to ASes and router rendezvous, PoPs observe less traffic due to the invisibility of traffic within ASes (intra-domain traffic). Moreover, the number of PoPs is small so that the points of monitoring are limited. Among the three, router rendezvous make a tradeoff. Their detection capabilities are comparable to PoPs' and they can observe intra-domain traffic with more monitoring points available, making detection more feasible.

\section{Discussions AND CONCLUSION}

For actual implementation of botnet detection in the Internet infrastructure, there remain a few challenges to be addressed. First, since our techniques are applied at the structure-level via graph analysis, they will also identify regular P2P file-sharing topologies. To avoid misclassifying such regular P2P networks as botnets, we can perform preprocessing such as flow filtering and clustering [9] based on known patterns of regular P2P networks such as the port numbers. Also, bots identified locally in edge networks are helpful as their presence in a communication graph makes other nodes suspicious as well, so our approach may need assistance from detection mechanisms at the edge to further confirm that a graph is indeed formed by a botnet. However, if the botnet is immersed into an existing regular P2P network, detecting it solely by graph analysis at the infrastructure level would be challenging and other information is thus needed for effective detection. Second, our models on the Internet infrastructure are abstracted from real-world datasets, so the accuracy depends on how well the datasets characterize the behavior and the state of the Internet, which could be error-prone. Moreover, some datasets may be outdated and may not reflect the current state of the Internet due to its fast-evolving nature. Lastly, in the presence of a huge traffic volume, some connections could not be captured due to sampling. For densely-connected topologies such as Kad, it may not be a problem. But for the modified Chord and simple ring's cases, it will complicate the detection. We plan to dig deeper into this issue.

To sum up, as P2P structures become a popular choice for recent botnets, especially large-scale ones, detection mechanisms have to keep up with this change and identify bots in an efficient and effective manner. In this paper, we propose detection of $\mathrm{P} 2 \mathrm{P}$ botnets at a high-level-the infrastructurelevel by analyzing their structural properties from a graph perspective. We find that detection at any of the three network components has its advantages and drawbacks. Overall, routerrendezvous-level detection is able to strike a balance between detection capability and feasibility. Also, a botnet needs to make a tradeoff between resilience and stealthiness.

\section{ACKNOWLEDGMENTS}

The work reported in this paper was supported in part by the ONR under Grant No. N000140911042 and the NSF under Grant No. CNS 0905143.

\section{REFERENCES}

[1] "aMule," http://www.amule.org/.

[2] "AS Names," http://bgp.potaroo.net/cidr/autnums.html.

[3] "PRIME SSF," https://www.primessf.net/bin/view/Public.

[4] J. R. Binkley and S. Singh, "An algorithm for anomaly-based botnet detection," in Proceedings of the 2nd conference on Steps to Reducing Unwanted Traffic on the Internet, 2006.

[5] C. R. Davis, S. Neville, J. M. Fernandez, J.-M. Robert, and J. McHugh, "Structured peer-to-peer overlay networks: Ideal botnets command and control infrastructures?" in Proceedings of ESORICS'08.

[6] G. Gu, R. Perdisci, J. Zhang, and W. Lee, "BotMiner: Clustering analysis of network traffic for protocol- and structure-independent botnet detection," in Proceedings of the USENIX Security Symposium, 2008.

[7] G. Gu, J. Zhang, and W. Lee, "Botsniffer: Detecting botnet command and control channels in network traffic," in Proc. of NDSS, 2008.

[8] D. Ha, G. Yan, S. Eidenbenz, and H. Ngo, "On the effectiveness of structural detection and defense against p2p-based botnets," in Proceedings of DSN 2009.

[9] M. Iliofotou, H. chul Kim, P. Pappu, M. Faloutsos, M. Mitzenmacher, and G. Varghese, "Graph-based p2p traffic classification at the internet backbone," in Proceedings of IEEE Global Internet Symposium, 2009.

[10] M. Iliofotou, P. Pappu, M. Faloutsos, M. Mitzenmacher, S. Singh, and G. Varghese, "Network monitoring using traffic dispersion graphs (tdgs)," in Proceedings of IMC 2007.

[11] M. Jelasity and V. Bilicki, "Towards automated detection of peer-to-peer botnets: On the limits of local approaches," in Proc. of LEET, 2009.

[12] P. Maymounkov and D. Mazieres, "Kademlia: A peer-to-peer information system based on the xor metric," in Proceedings of IPTPS, 2001.

[13] T. N. McPherson and M. J. Brown, "Estimating daytime and night-time population distributions in u.s. cities for emergency response activities," The American Meteorological Society.

[14] S. Nagaraja, P. Mittal, C.-Y. Hong, M. Caesar, and N. Borisov, "Botgrep: Finding p2p bots with structured graph analysis," in Proceedings of 19th USENIX Security Symposium, 2010.

[15] J. Qiu and L. Gao, "As path inference by exploiting known as paths," in Proceedings of IEEE GLOBECOM, 2005.

[16] A. H. Rasti, R. Rejaie, and W. Willinger, "Characterizing the global impact of p2p overlays on the as-level underlay," in Proceedings of PAM 2010.

[17] I. Stoica, R. Morris, D. Karger, M. F. Kaashoek, and H. Balakrishnan, "Chord: A scalable peer-to-peer lookup service for internet applications," in Proceedings of ACM SIGCOMM, 2001.

[18] R. Waupotitsch, S. Eidenbenz, J. Smith, and L. Kroc, "Multi-scale integrated information and telecommunications system (MIITS): First results from a large-scale end-to-end network simulator," in Proceedings of the Winter Simulation Conference, 2006.

[19] G. Yan, S. Eidenbenz, S. Thulasidasan, P. Datta, and V. Ramaswamy, "Criticality analysis of internet infrastructure," Computer Networks, vol. 54 , no. 7, 2010.

[20] Y. Zeng, X. Hu, and K. G. Shin, "Detection of botnets using combined host- and network-level information," in Proceedings of DSN, 2010. 